\title{
Contrast-Invariant Registration of Cardiac and Renal MR Perfusion Images
}

\author{
Ying Sun ${ }^{1}$, Marie-Pierre Jolly², and José M.F. Moura ${ }^{1}$ \\ 1 Department of Electrical and Computer Engineering, Carnegie Mellon University, \\ Pittsburgh, PA 15213, USA \\ ${ }^{2}$ Department of Imaging and Visualization, Siemens Corporate Research, Inc. \\ Princeton, NJ 08540, USA
}

\begin{abstract}
Automatic registration of dynamic MR perfusion images is a challenging task due to the rapid changes of the image contrast caused by the wash-in and wash-out of the contrast agent. In this paper we introduce a contrast-invariant similarity metric and propose a common framework to perform affine registration on both cardiac and renal MR perfusion images. First, large-scale translational motion is identified by tracking a selected region of interest with integer pixel shifts. Then, we estimate the affine transformation of the organ for each frame. We have tested the proposed algorithm on real cardiac and renal MR perfusion scans and obtained encouraging registration results.
\end{abstract}

\section{Introduction}

Dynamic perfusion magnetic resonance imaging (MRI) has demonstrated great potential for diagnosing cardiovascular and renovascular diseases [1] [2]. In dynamic perfusion MRI, the organ under study is scanned repeatedly and rapidly following a bolus injection of a contrast agent. Changes in pixel intensity corresponding to the same tissue across the image sequence provide valuable functional information about the organ being imaged. However, perfusion MR image sequences suffer from motion induced by patient breathing during acquisition. Therefore, registration must be performed on time-series images to ensure the correspondence of anatomical structures in different frames. Due to the vast amounts of data acquired in dynamic perfusion MRI studies (on average, over 100 images per scan), automatic registration is strongly desirable. This paper focuses on automatic registration of cardiac and renal MR perfusion images.

Goal: Given a sequence of perfusion MR images for the heart or the kidney, we want to solve the registration problem: establish the appropriate correspondence between every pixel in the region of interest in each frame of the sequence.

Difficulties: This is difficult and standard block matching techniques do not work because the intensity at the same physical location changes across the MR image sequence due to the wash-in and wash-out of the contrast agent. To the best of our knowledge, there has been limited work on image registration to address these difficulties. An image registration algorithm that utilizes the maximization of mutual information has recently been proposed for cardiac MR 


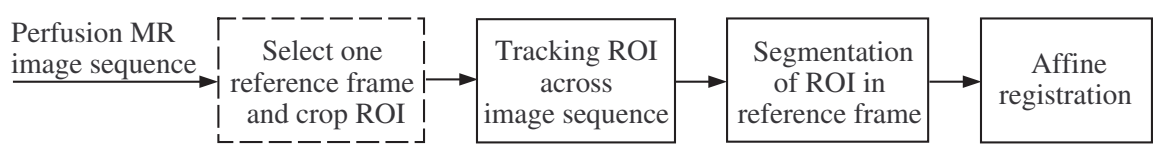

Fig. 1. The block diagram of the proposed registration algorithm

perfusion data 3]. Several methods have been developed for registration of renal MR perfusion images [4] [5] 6]. These methods all require manually drawn contours in one time frame to obtain a mask or a model; this model is then used to propagate the contours to other frames in the image sequence.

Our approach: We avoid manually drawn contours and only require the user to crop a rectangular region of interest (ROI). Since translation is the dominant motion caused by breathing, we divide the registration problem into two steps: large translation motion followed by affine transformation. The detailed sequence of steps is the following.

(1) Choose a reference frame and define a bounding box for the ROI;

(2) Compute the edge map in the bounding box of the reference frame and in the search window of other frames in the MR image sequence;

(3) Determine large-scale translation motion of the ROI by maximizing a contrast-invariant similarity metric between the current and the reference frames;

(4) Obtain the contours that delineate the boundaries of the organ in the reference frame through the segmentation of a difference image;

(5) Propagate the segmentation results to other frames by searching for the affine transformations that best match these frames to the reference frame.

Except for the first step, all other steps are automatic. The experimental results with real patient data show that by exploiting the invariance of the similarity metric our algorithm provides very good registration results.

\section{Method}

From the application point of view, it is reasonable to ask the user to select from the sequence of images one that has a good contrast and then crop an ROI in this selected frame. Fig. 11ilustrates the three main stages of the algorithm: tracking the ROI across the image sequence, segmentation of the ROI in the reference frame, and affine registration. Except for the segmentation block that is explained elsewhere, these stages are described in detail in the following sections with experiments on real MR perfusion patient datasets .

\subsection{Tracking the ROI Across the Image Sequence}

Given an ROI in one frame, the goal of this stage is to find the best match to the selected ROI in other frames. At this stage, we assume that the motion is reduced 
to translation with integer pixel shifts. Tracking the ROI is achieved by simple template matching. The key observation is that the orientation of the edges along tissue boundaries are always parallel across the image sequence, despite the fact that the relative intensities between tissues vary with time. Therefore, we choose the template defined by the orientation of the image gradient.

In our formulation, the image on which the ROI is manually cropped is called the reference frame. Let $\theta_{r}(x, y)$ and $M_{r}(x, y)$ stand for respectively the direction and the magnitude of the image gradient at pixel $(x, y)$ in the reference frame; we obtain $\theta_{r}$ and $M_{r}$ using a Sobel edge detector, [7]. We denote the set of pixels in the ROI as $\mathcal{R}=\left\{(x, y) \mid x_{a} \leq x \leq x_{b}, y_{a} \leq y \leq y_{b}\right\}$, where $\left(x_{a}, y_{a}\right)$ and $\left(x_{b}, y_{b}\right)$ are the two diagonal points that specify the bounding box of the ROI. Let $\theta_{c}(x, y)$ denote the edge orientation and $M_{c}(x, y)$ the edge magnitude at pixel $(x, y)$ in the current frame. For each offset pair $(d x, d y)$, we define the angle difference $\Delta \theta(x, y ; d x, d y)$ and a weight function $w(x, y ; d x, d y)$ by

$$
\begin{aligned}
\Delta \theta(x, y ; d x, d y) & =\theta_{c}(x+d x, y+d y)-\theta_{r}(x, y), \\
w(x, y ; d x, d y) & =\frac{M_{c}(x+d x, y+d y) M_{r}(x, y)}{\sum_{(x, y) \in \mathcal{R}} M_{c}(x+d x, y+d y) M_{r}(x, y)} .
\end{aligned}
$$

We introduce a similarity metric:

$$
S(d x, d y)=\sum_{(x, y) \in \mathcal{R}} w(x, y ; d x, d y) \cos (2 \Delta \theta(x, y ; d x, d y)) .
$$

Note that $S(d x, d y)$ is the weighted average of the values of $\cos (2 \Delta \theta)$ over the ROI, and its value lies in the interval of $[-1,1]$. We use the cosine of the double angle to handle contrast inversions that commonly occur in perfusion studies. For instance, in a renal MR perfusion scan, see the first image in the bottom row in Fig. 2 the kidney is relatively darker compared to the surrounding tissues before the wash-in of the contrast agent; it becomes relatively brighter after the wash-in of the contrast agent as shown in the second image in the bottom row in Fig. 2. In addition, we choose the weight function as the normalized product of the edge magnitudes because it is desirable for the ROI to be attracted to strong edges in the current frame that are mostly parallel to the strong edges in the reference frame. The proposed similarity metric is invariant to rapidly changing image contrast, in the sense that its value is insensitive to changes in the contrast as long as the edge orientations are nearly parallel to those of the template. The integer shifts $\left(d x^{*}, d y^{*}\right)$ that maximize $S$ are determined by exploring all possible solutions $(d x, d y)$ over a reasonable search space. It is important to point out that the value of $S\left(d x^{*}, d y^{*}\right)$ also plays a role as a confidence measure. To improve the robustness of the algorithm, we use both the previous frame and the reference frame as templates. The algorithm then chooses the match with maximum similarity metric.

We display in Fig. 2 selected frames from a renal (top row) and a cardiac (bottom row) MR perfusion scan, respectively. In cardiac perfusion, the contrast agent passes through the right ventricle to the left ventricle and then perfuses into the myocardium. Similarly, the intensity of the kidney increases as the contrast 

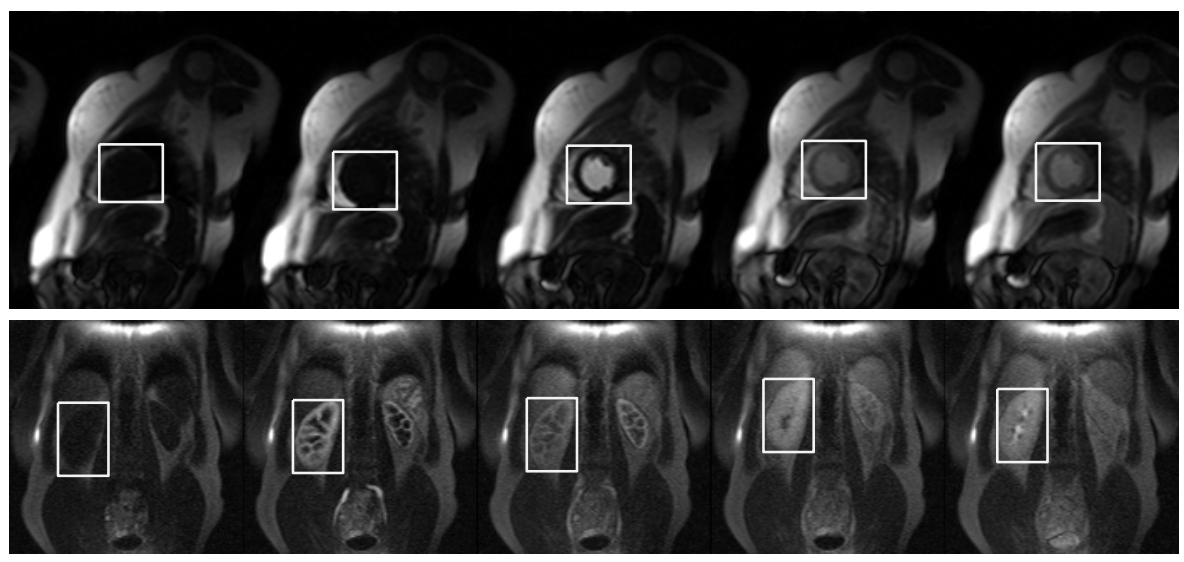

Fig. 2. Results obtained by tracking the ROI with integer pixel shifts on selected frames from a cardiac (top) and a renal (bottom) MR perfusion image sequence.

agent perfuses into the cortex, the medulla, and other structures of the kidney. To illustrate the performance of the proposed tracking algorithm, we shift the bounding box of the ROI to the best match location in each frame, see Fig. 2] Despite the rapidly changing image contrast and the fact that translational motion between two adjacent frames can be considerably large, the algorithm reliably tracks the selected ROI across the image sequence for both cardiac and renal perfusion studies, with minor tracking error in frames that lack strong edges. To further improve the accuracy of the registration results, we propose to estimate the local affine transformation of the heart or kidney by incorporating the knowledge of the contours delineating organ boundaries in the reference frame. This is considered in subsection 2.3 .

\subsection{Segmentation of the ROI}

The purpose of this stage is to identify the boundaries of the organ in the reference frame, based on roughly registered ROI sequence resulting from the previous subsection. It has been demonstrated that myocardial boundaries can be detected by segmenting a subtraction image, in which the myocardium is accentuated [8]. Fig. 3(c) displays the image obtained by subtracting Fig. 3(a) from Fig. 3(b). Detecting the boundaries of the left ventricle becomes a less challenging problem, to which we can apply many available segmentation algorithms [8] 9] 10] 11. We take an energy minimization approach and propose a customized energy functional to overcome some unique problems that arise in our particular application. To take into account the anatomical constraint that the distance between the endocardium and the epicardium is relatively constant, we borrow the idea of coupled propagation of two cardiac contours [9]. Since the emphasis of this paper is placed on registration, we will directly present the segmentation results with no further explanation. The detected endocardium and epicardium are delineated respectively using bright contours on top of the ref- 


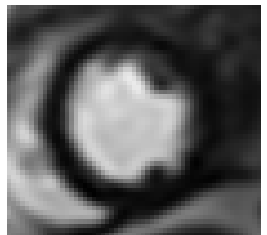

(a)

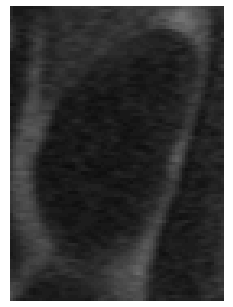

(e)

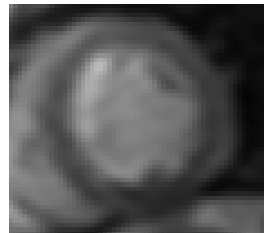

(b)

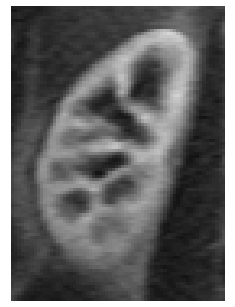

(f)

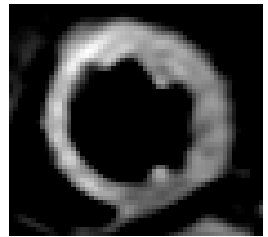

(c)

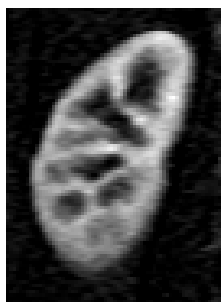

$(\mathrm{g})$

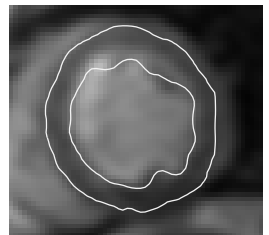

(d)

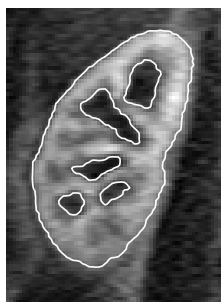

(h)

Fig. 3. Segmentation results of roughly registered images.

erence frame, see Fig. 3 (d). Although this is an approximate segmentation, it provides us with enough shape priors to refine the template for affine registration.

Similar to the case of cardiac MR perfusion data, the subtraction between Figs. 3(e) and (f) results in an enhanced image for the renal MR perfusion scan as shown in Fig. [3(g). We apply the level set method described in 11 to extract the renal cortex. This is an energy minimization based segmentation method. It assumes that the image is formed by two regions of approximatively piecewise constant intensities of distinct values [11. It can be seen in Fig. 3(g) that the assumption is valid; the image contains a bright object to be detected in a dark background. The segmentation results are overlaid on top of the reference frame in Fig. 3(h). As shown, the outer boundary of the kidney is well delineated. These results demonstrate that the boundaries of the organ (heart or kidney) in the reference frame can be identified.

\subsection{Affine registration}

We have compensated for integer pixel shifts of the ROI and obtained the contours that delineate the boundaries of the organ in the reference frame. Next, we propagate the segmentation results to other frames by estimating the affine transformation of the organ in each frame.

The affine transformation includes translation, rotation, scaling and shearing. In $2 \mathrm{D}$, the affine transformation between $(x, y)$ and $\left(x^{\prime}, y^{\prime}\right)$ is given by

$$
\left[\begin{array}{l}
x^{\prime} \\
y^{\prime}
\end{array}\right]=\mathbf{A}\left[\begin{array}{l}
x \\
y
\end{array}\right]+\mathbf{t},
$$

where $\mathbf{A}$ is a $2 \times 2$ invertible matrix that defines rotation, shear, and scale, and $\mathbf{t}$ is a $2 \times 1$ translation vector. 

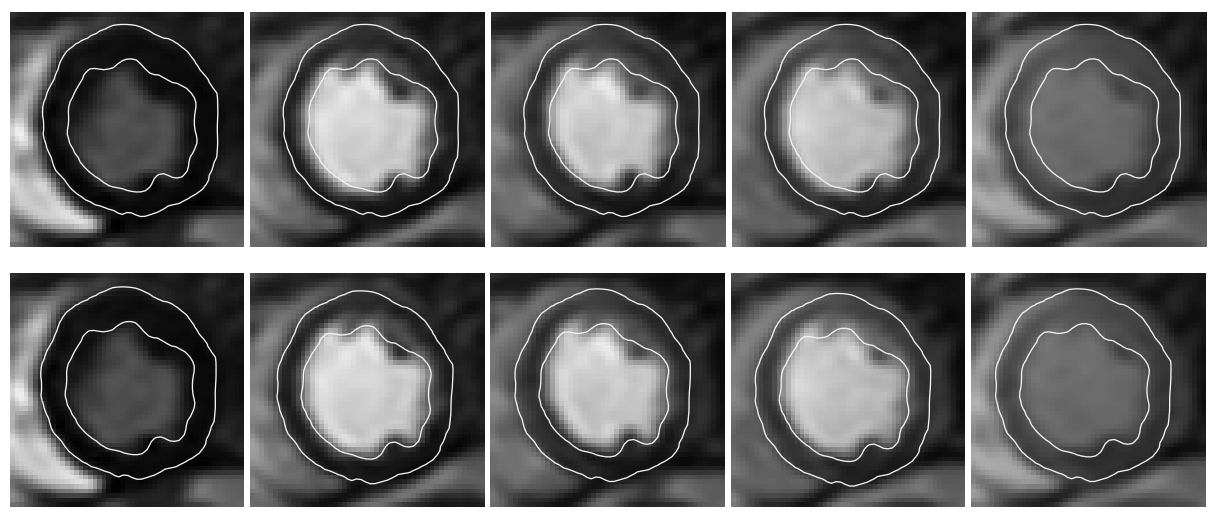

Fig. 4. Registration results for representative frames from a real patient cardiac MR perfusion scan. The top row shows the segmentation results obtained in Section 2.2 with the contours overlaid on the registered ROI. The bottom row shows the transformed contours after applying affine registration.

The segmentation results obtained in the previous step make it possible to refine the template by ignoring irrelevant edge information. Let $\mathcal{L} \subset \mathcal{R}$ denote the set of pixels in the ROI corresponding to pixels lying on the boundaries of the organ and their nearest neighbors under a second order neighborhood system. Let $\theta_{c}^{\prime}(x, y ; \mathbf{A}, \mathbf{t})$ and $M_{c}^{\prime}(x, y ; \mathbf{A}, \mathbf{t})$ denote the corresponding direction and magnitude of the image gradient in the current frame under the affine transformation defined by $\mathbf{A}$ and $\mathbf{t}$. The goal of the affine registration is to find the affine transformation $(\mathbf{A}, \mathbf{t})$ that maximizes the following similarity metric for the current image

$$
S^{\prime}(\mathbf{A}, \mathbf{t})=\sum_{(x, y) \in \mathcal{L}} w^{\prime}(x, y ; \mathbf{A}, \mathbf{t}) \cos \left(2 \Delta \theta^{\prime}(x, y ; \mathbf{A}, \mathbf{t})\right),
$$

where $\Delta \theta^{\prime}(x, y ; \mathbf{A}, \mathbf{t})$ and $w^{\prime}(x, y ; \mathbf{A}, \mathbf{t})$ are computed respectively by

$$
\begin{aligned}
\Delta \theta^{\prime}(x, y ; \mathbf{A}, \mathbf{t}) & =\theta_{c}^{\prime}(x, y ; \mathbf{A}, \mathbf{t})-\theta_{r}(x, y), \\
w^{\prime}(x, y ; \mathbf{A}, \mathbf{t}) & =\frac{M_{c}^{\prime}(x, y ; \mathbf{A}, \mathbf{t}) M_{r}(x, y)}{\sum_{(x, y) \in \mathcal{L}} M_{c}^{\prime}(x, y ; \mathbf{A}, \mathbf{t}) M_{r}(x, y)} .
\end{aligned}
$$

In the special case where the motion of the organ is highly constrained and approximately rigid, we can reduce the number of degrees of freedom in the affine transform by restricting the motion to rotation and translation. In our experiments, we adopt the following measures: (1) we keep the scaling fixed and let the rotation vary between -5 to 5 degrees in 1 degree steps; (2) the translation along either dimension is constrained between -2 to 2 pixels; (3) we construct a bank of templates for each combination of rotation and translation; and (4) we search for the best template that results in the largest similarity metric between the current frame and the reference frame, over the constrained parameter space. This algorithm is fast and insensitive to noise. Although not described here, the algorithm can be extended to maximizing $S^{\prime}(\mathbf{A}, \mathbf{t})$ under affine transformation through a gradient descent method [12]. 

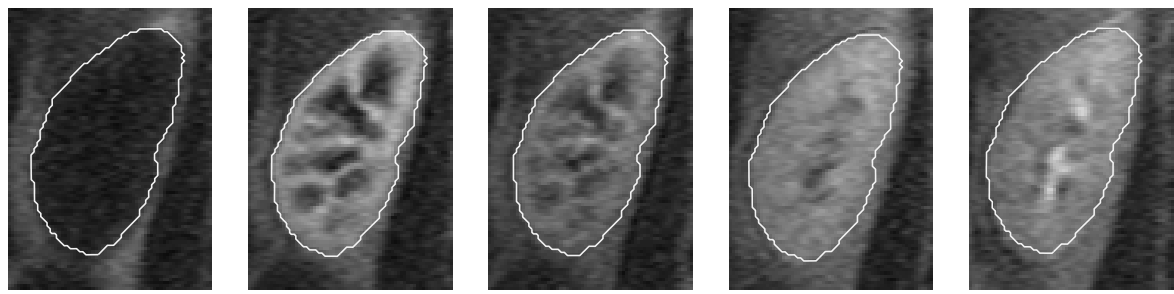

Fig. 5. Registration results for selected frames from a renal MR perfusion scan.

\section{Results}

Our algorithm has been tested on 15 cardiac and 12 renal MR perfusion datasets of real patients. Each dataset contains 3 or 4 slices. The images were acquired on Siemens Sonata MR scanners following bolus injection of Gd-DTPA contrast agent. In most cases, the image matrix is $256 \times 256$ pixels. The number of frames in each image sequence ranges from 50 to 350.

For all the data sets in our study, the registration results were qualitatively validated with visual analysis by displaying the registered image sequence in a movie mode. Using our ROI tracking algorithm, we are able to track the ROI reliably in all the sequences robustly, with a maximum tracking error less than 2 pixels in both directions. Furthermore, our experimental results show that affine registration helps improve the accuracy of the registration greatly. Fig. 4 compares the results obtained before and after applying affine registration for a real cardiac MR perfusion scan. As shown, the contours in the top row of images, before affine registration, do not lie exactly at the boundaries of the left ventricle. This is easily seen at the bottom left of each image where the edge information is relatively strong. On the other hand, the contours in the bottom row of images, after affine registration, delineate well the boundaries of the left ventricle. In particular, the accuracy of the estimated rotation can be visually validated by looking at the papillary muscle at the bottom of each image.

To illustrate the performance of the registration algorithm with renal MR perfusion data, we present the results for a real patient in Fig. 5. We have also validated the registration results quantitatively for a renal perfusion MR scan of 150 images, by comparing the estimated translation vector at each frame with a "gold standard," i.e., pixel shifts obtained manually. The error size is less than one pixel in each direction for more than $95 \%$ of the frames. These results strongly suggest that the motion caused by breathing can be successfully compensated using the registration algorithm presented here.

\section{Conclusion}

The paper presents a common framework for the registration of dynamic cardiac and renal MR perfusion images. The algorithm exploits image features that are invariant to a rapidly changing contrast and utilizes image segmentation results for the construction of templates. We have obtained encouraging registration 
results with real patient datasets. As future work, we plan to segment different anatomical structures within the ROI based on their distinct dynamics of the first-pass signal, i.e., the pixel intensity-time curve [13.

Acknowledgements. The authors would like to thank Niels Oesingmann from Siemens Medical Systems MR Division, Erlangen, Germany, and Stefan Schoenberg from the Institute of Clinical Radiology, Klinikum Grosshadern, Munich, Germany, for providing cardiac and renal MR perfusion data. The first and third authors acknowledge the support from NIH grants (P41EB001977 and R01EB/AI-00318) and from a Siemens research grant.

\section{References}

1. E. Nagel et al. Magnetic resonance perfusion measurements for the noninvasive detection of coronary artery disease. Circulation, 108(4):432-437, July, 2003.

2. S.J. Gandy et al. Dynamic MRI contrast enhancement of renal cortex: a functional assessment of renovascular disease in patients with renal artery stenosis. J Magn Reson Imaging, 18(4):461-6, Oct, 2003.

3. R. Bansal and G. Funka-Lea. Integrated image registration for cardiac MR perfusion data. MICCAI(1) 2002:659-666.

4. G. Gerig, R. Kikinis, W. Kuoni, G.K. van Schulthess, and O. Kübler. Semiautomated ROI analysis in dynamic MRI-studies: Part I: image analysis tools for automatic correction of organ displacements. IEEE Trans. Image Processing, 11(2):221232, 1992.

5. E.L.W. Giele et al. Movement correction of the kidney in dynamic MRI scans using FFT phase difference movement detection. J. Magn Reson Imaging, 14:74149, 2001.

6. P.J. Yim et al. Registration of time-series contrast enhanced magnetic resonance images for renography. Proc. 14th IEEE Symp. Computer-Based Medical Systems, pp. 516-520, 2001.

7. K.R. Castleman. Digital Image Processing. Prentice Hall, 1996.

8. L. Spreeuwers and M. Breeuwer. Automatic detection of myocardial boundaries in MR cardio perfusion images. MICCAI 2001:1228-1231.

9. N. Paragios. A variational approach for the segmentation of the left ventricle in MR cardiac images. IEEE Workshop on Variational and Level Set Methods in Computer Vision, Vancouver, Canada, pp.153-160, July 2001.

10. M.-P. Jolly. Combining edge, region, and shape information to segment the left ventricle in cardiac MR images. Proc. Medical Image Computing and ComputerAssisted Intervention, Utrecht, The Netherlands, pp.482-490, Oct 2001.

11. T. F. Chan and L. A. Vese. Active contours without edges. IEEE Trans. Image Processing, 10(2):266-77, 2001.

12. A. Yezzi, L. Zollei, and T. Kapur. A variational framework for joint segmentation and registration. IEEE CVPR-MMBIA, 2001.

13. Y. Sun, J. M. F. Moura, D. Yang, Q. Ye, and C. Ho. Kidney segmentation in MRI sequences using temporal dynamics. Proc. 2002 IEEE Int. Symp. Biomedical Imaging, pp.98-101, Washington, D.C., July 2002. 